\title{
Assessment of the burden of human African trypanosomiasis by rapid participatory appraisal in three high-risk villages in Urambo District, Northwest Tanzania
}

\author{
Reid $\mathrm{H}^{1}$, Kibona $\mathrm{S}^{2}$, Rodney $\mathrm{A}^{1}$, McPherson $\mathrm{B}^{1}$, Sindato $\mathrm{C}^{2}$, Malele $\mathrm{I}^{4}$, Kinung'hi $\mathrm{S}^{3}$, Jennaway $\mathrm{M}^{1}$, \\ Changalucha $\mathrm{J}^{3}$, Blake $\mathrm{B}^{1}$, *Vallely $\mathrm{A}^{1,3}$.
}

1. Australian Centre for International and Tropical Health, School of Population Health, University of Queensland, Herston, Brisbane, QLD 4006, Australia

2. National Institute for Medical Research, Tabora Centre, PO Box, Tabora, Tanzania

3. National Institute for Medical Research, Mwanza Centre, PO Box 1462, Mwanza, Tanzania

4. Tsetse \& Trypanosomiasis Research Institute (TTRI), PO Box 1026 Tanga, Tanzania

\begin{abstract}
Background: The public health and socio-economic burden of Human African Trypanosomiasis (HAT) in East Africa is not well documented. Understanding the epidemiology and impact of HAT in such settings is difficult due to a lack of robust surveillance and reporting systems, restricting evidence-based policy development and contributing to the continued neglect of this disease.

Objective: To investigate the burden of HAT in Urambo District, Tanzania in order to inform future public health policy. Methods: A rapid participatory appraisal (RPA) using a combination of qualitative and quantitative methods was conducted, that included key informant interviews, hospital record analysis, and tools adapted from participatory learning and action. Results: Three villages adjacent to Ugala Game Reserve appeared to be the most affected. High levels of under-reporting were noted due to a lack of diagnostic tools at peripheral health care facilities and limited access to specialist services. Community stakeholders perceived the health and socio-economic burden of HAT to be similar to that of malaria.

Conclusion: The burden of HAT in remote rural communities is difficult to capture through routine surveillance systems alone. The RPA represents an efficient mechanism for engaging communities in public health action for trypanosomiasis control in northwest Tanzania.
\end{abstract}

Keywords: trypanosomiasis, disease burden, rapid appraisal, community, Tanzania

African Health Sciences 2012; 12(2): 104 - 113 http://dx.doi.org/10.4314/ahs.v12i2.5

\section{Introduction}

Human African Trypanosomiasis (HAT), commonly known as sleeping sickness, is a zoonotic disease due to infection with the parasites Trypanosoma brucei gambiense (T.b.gambiense) or Trypanosoma brucei rhodesiense (T.b. rhodesiense) that are transmitted by insect vectors of the genus Glossina (tsetse flies). There are two recognized stages in the clinical presentation of HAT: an early hemolymphatic stage which is associated with a febrile illness; and a late encephalitic stage, which develops after parasites have invaded the

\begin{tabular}{l}
\hline *Correspondence author: \\
Dr Andrew Vallely \\
Associate Professor \\
Public Health Interventions Research Group, Kirby \\
Institute \\
University of New South Wales \\
45 Beach Street, Sydney \\
NSW 2034, Australia \\
E-mail: avallely@kirby.unsw.edu.au \\
\hline
\end{tabular}

cerebrospinal fluid (CSF) ${ }^{1}$. Infection with T.b. rhodesiense usually manifests as an acute illness, with parasites invading the Central Nervous System (CNS) a few months after initial infection, while T.b. gambiense is typically a slower, chronic illness, with invasion of the CNS months to years after initial infection ${ }^{1}$.

The 1996 Global Burden of Disease Report ${ }^{2}$ was the first attempt to quantify the burden of HAT. An important limitation of these estimates however, was the failure to distinguish between T.b. gambiense and T.b. rhodesiense which, as detailed above, have differing disease progressions and sequale. Recently, Fevre et $\mathrm{al}^{3}$ provided the first estimates of disability weightings for the specific stages of T.b. rhodesiense disease progression by exploring HAT cases in a transmission foci in Uganda. In addition to the burden of morbidity and mortality resulting from HAT, there is also a paucity of information regarding the social and economic burden of T. b. rhodesiense with just a handful of studies investigating these issues. 
Studies in Uganda ${ }^{4}$ and the Democratic Republic of Congo ${ }^{5}$ both found that the indirect costs of HAT treatment to the household are substantial.

The HAT was almost eliminated from Africa in the 1960's following the success of control programs in a number of endemic countries. The instability of the post-colonial period however, saw many programs discontinued and the subsequent reemergence of the disease ${ }^{6-7}$. In Tanzania, communities adjacent to the Ugala Game Reserve in Urambo District in the northwest of the country have recently been identified by the Tanzania National Institute for Medical Research (NIMR) as high transmission foci of $T$. $b$. rhodesiense infection following reports of increasing numbers of HAT cases from 2003 onwards. Case data from this area for 2004 has been used to estimate disability adjusted life years (DALYs) for T. b. rhodesiense $e^{8}$.

The aim of this study was to assess how HAT is perceived as a public health problem in three highly affected villages in Urambo District using a mixedmethods, participatory research approach, and to use key findings to inform the public health response to HAT in affected villages.

\section{Methods}

A Rapid Participatory Appraisal (RPA) was conducted over a three-month period in mid-2008. Qualitative tools were adapted from Participatory Learning and Action (PLA) techniques, successfully used in a variety of locally-appropriate, communitybased interventions in other settings ${ }^{9-10}$.

\section{Table 1: List of key informants (KIIs)}

An iterative research process was adopted to facilitate understanding of the disease in this sociocultural context; to investigate how communities interpret health and disease; and to explore how local stakeholders could help mitigate the disease burden.

Purposive snowball sampling was used to identify a representative sample of key informants who included National Institute of Medical Research (NIMR) researchers, Ministry of Health and Social Welfare (MoHSW) and District Council officials, Ministry of Livestock Development and Fisheries (MoLDF) officers, hospital administrators and laboratory technicians, village leaders/chairpersons, village influential women, HAT sufferers (past and present), semi-nomadic tribesmen, traditional healers, dispensary workers, and those involved in tsetse control activities.

Semi-structured Key Iinformant Interviews (KIIs) were conducted $(\mathrm{n}=38)$ until the point of thematic saturation (table 1). The KIIs were questioned on their knowledge of HAT and perceptions concerning impact of the disease and asked to provide information specific to their position. For example, traditional healers were asked more comprehensive questions on the diagnosis and treatment of HAT and perceptions of health and disease in general, while village leaders were asked to provide more detailed information of the village itself. Qualitative data from KIIs were analysed at the University of Queensland using NVivo 7 software (QSR International Ltd, Australia).

\begin{tabular}{lll}
\hline Level & Key informant & Number \\
\hline Higher level & National Institute of Medical Research (NIMR) Laboratory technicians and & 3 \\
& research scientists & \\
& Ministry of Health informants & 2 \\
& District Council Development officers & 1 \\
& Ministry of Livestock development and fisheries officers & 2 \\
& Kaliua Health Centre staff & 2 \\
Village level & Village Chairperson/Executive officer & 4 \\
& Village Influential Woman/Secretary of Women's group & 3 \\
& Case studies & 6 \\
& High risk individuals & 6 \\
& General population & 4 \\
& Sukuma tribe herdsman & 1 \\
& Traditional healer & 2 \\
& Dispensary worker & 1 \\
& Chairperson of Tseste control committee & 1 \\
\hline
\end{tabular}


Participatory tools were used to investigate how villages prioritise health issues affecting their lives. A listing and ranking exercise was conducted in samesex groups of 6-7 villagers who were asked to generate a list of priority health issues that most affected them, their families, and the community in most terms of treatment, suffering and cost. Individually, participants were then asked to distribute 13 beans between the health issues reflecting their personal opinions as to which created a greater burden (e.g. 5 beans next to the most important disease, 3 beans to the second most important issue and 1-2 beans to other issues of minor importance). Once all participants had voted in this way, summary scores were obtained for each health issue and a ranked list of diseases compiled and discussed within the group. Pair-wise matrices were then developed by group members using the ranked list of health priorities and a hypothetical scenario presented: given two diseases and the resources to remove just one disease, which disease would the group rather remove?

Participatory community mapping was conducted in order to promote shared understanding between researchers and participants regarding the roles, responsibilities and duties associated with daily village life; the community's reliance on the local environment; and subsequently the degree to which villagers were exposed to tsetse habitats. Small-scale maps of each of the three villages were jointly developed by senior village representatives and the research team.

Information on confirmed HAT cases from 2000 onwards was collected at Kaliua Health Centre (KHC), which provided the most complete source of data in the District.

Figure 1: Map of Tanzania indicating study area (Urambo district)

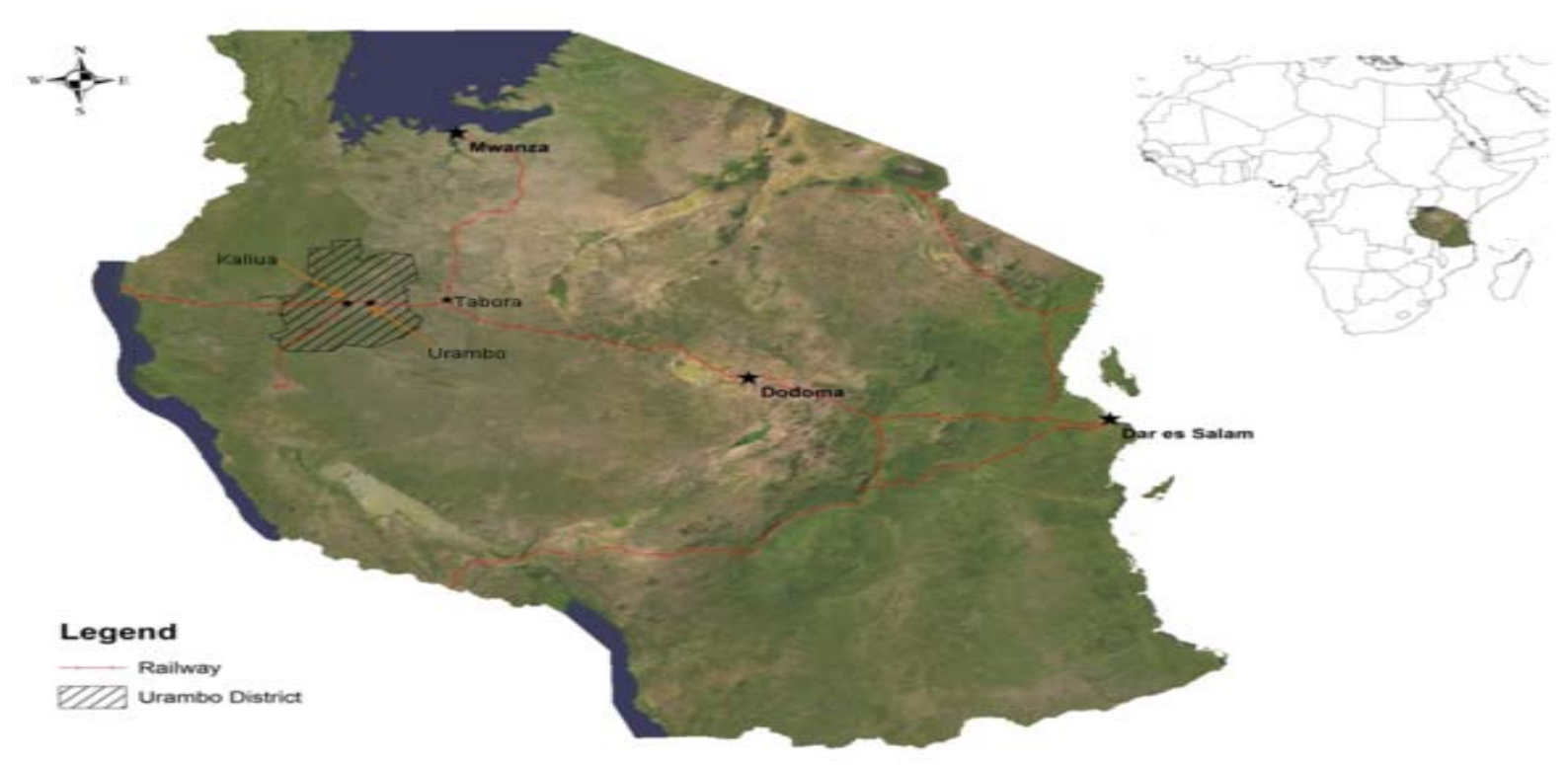

Cases were identified by origin, sex and age from 2003 and also by month from 2004. Case numbers were used to estimate crude incidence using population estimates derived from the 2002 National Census and adjusted to the growth rate for Tabora Region ${ }^{11}$. Incidence estimates were used to make comparisons between villages and the district as a whole, and to highlight the highly-focal nature of HAT. Only cases that could be identified by village of residence (2003-2007) were included in these measures. Case-Fatality Rates (CFR) were calculated from 2002 when KHC first began treating HAT patients.

\section{Ethical issues}

Ethical approval for this research was obtained from the National Medical Research Coordinating Committee in Tanzania and the University of Queensland, Australia. All study participants provided written informed consent (or a witnessed thumbprint) prior to enrolment into the study.

\section{Results}

\section{Study area}

From KHC hospital records, three villages (Lumbe, Kangeme, and Usinga), all from Urambo District, were identified as having significant numbers of HAT cases, and selected for further study. The three villages all lie along the Kaliua to Mpanda rail-line, with transport between villages mostly by bicycle. A bus service operates between Kaliua and the villages during the dry season but not during the wet season when the road becomes impassable. Villages are surrounded by miombo woodland, a desirable habitat for tsetse flies. 


\section{Epidemiology of HAT in Urambo District}

At KHC, 396 cases of HAT were reported between 2003 and 2007: 53\% $(n=212)$ were thought to have contracted HAT in Urambo district (table 2); while
$44 \%$ and $2 \%$ of the remaining cases were thought to have contracted the disease in neighbouring Mpanda district and Kigoma region respectively.

Table 2: Reported cases and cumulative incidence per 100,000 population/year of HAT in study villages and Urambo district, 2003-2007

\begin{tabular}{|c|c|c|c|c|c|c|c|c|c|c|c|c|c|}
\hline \multirow[t]{2}{*}{ Village } & \multicolumn{2}{|c|}{2003} & \multicolumn{2}{|c|}{2004} & \multicolumn{2}{|c|}{2005} & \multicolumn{2}{|c|}{2006} & \multicolumn{2}{|c|}{2007} & \multicolumn{2}{|c|}{ Total } & \multirow{2}{*}{$\begin{array}{l}\text { RR } \\
2003-07\end{array}$} \\
\hline & $\mathrm{n}$ & CI & $\mathrm{n}$ & $\mathrm{CI}$ & $\mathbf{n}$ & CI & $\mathrm{n}$ & CI & $\mathbf{n}$ & $\mathrm{CI}$ & $\mathrm{n}$ & CI & \\
\hline Lumbe & 22 & 335 & 34 & 500 & 10 & 142 & 3 & 41 & 15 & 198 & 84 & 256 & 23 \\
\hline Kangeme & 25 & 834 & 15 & 483 & 13 & 404 & 6 & 180 & 6 & 174 & 65 & 434 & 39 \\
\hline Usinga & 7 & 772 & 8 & 851 & 3 & 308 & 2 & 198 & 4 & 383 & 24 & 530 & 48 \\
\hline Total & 75 & 20 & 64 & 16 & 35 & 9 & 12 & 3 & 26 & 6 & 212 & 11 & 1 \\
\hline
\end{tabular}

The peak in confirmed cases in 2003-2004 was thought to reflect an increased awareness of HAT in the community following church and government education campaigns. Key informants agreed that before 2003, HAT was not well known and was likely to have been mistaken for malaria, HIV or thought to be due to sorcery. The downward trend observed after 2004, also observed by Sindato et $\mathrm{al}^{12}$., was felt due to diminished tsetse fly numbers following the introduction of insecticide treated targets. The slight increase in incidence in 2007 was considered to be due to renewed emphasis on active case-finding by a microscopist from KHC.

Of the 396 cases diagnosed at KHC during the period 2003-2007, 264 were males (67\%) and 132 were females (33\%), 97\% were adults and 3\% were children under 13 years. Of the total of 12 cases reported in children, eight were among girls and four among boys. Informants from the MoHSW and KHC estimated that less than $50 \%$ of those contracting HAT in the district are diagnosed. It was not clear however, how this estimate was obtained.

Of the 377 people treated between 2003 and 2007 at KHC, 76 cases $(20 \%)$ received the earlystage drug, suramin, and 301 cases $(80 \%)$ the more toxic, second stage-drug, melarsoprol. From 20022007, there were 19 hospital-reported deaths at KHC, representing a case-fatality rate (CFR) of 5.0\% for those on treatment. Of the hospital-reported fatalities, it was not documented whether patients were receiving treatment with suramin for stage one disease, or melarsoprol for second stage disease. It was not possible using hospital records to differentiate deaths due to drug-related toxicity from those arising as a result of the complications of latestage infection.

African Health Sciences Vol 12 No 2 June 2012

\section{Perceptions of the burden and impact of HAT}

There were marked differences between the opinions of secondary stakeholders (e.g. from MoHSW, NIMR, VIC) and village-level primary stakeholders regarding the community burden of HAT. Secondary stakeholders felt that the comparative burden of HAT was small and the relative incidence across the District low and therefore that HAT was not a high priority for funding or public health intervention. The research team noted however, that several informants did not have specific knowledge pertaining to the treatment of HAT, the long recovery phase and the socio-economic impact on affected households.

Within affected communities, interviews and participatory group activities revealed a perception that HAT creates a significant burden in terms of disease morbidity and mortality experienced by the patient and social and economic costs to patients and their families. When asked to consider the cumulative health burden of all the diseases/ailments affecting each village, listing and ranking activities (table 3) revealed that HAT is perceived to contribute a similar, if not greater burden than malaria, which is widely recognized as one of the most important causes of mortality and morbidity in Tanzania ${ }^{13}$.

Within each village, participants perceived both HAT and malaria as each representing around one third of the total disease burden, with the final third comprising a combination of several other diseases, including intestinal worms, tuberculosis, skin conditions and diarrhoea. Pair-wise ranking confirmed the importance of both HAT and malaria at the village level (table 3). 


\begin{tabular}{|c|c|c|c|c|c|c|c|}
\hline \multicolumn{8}{|c|}{ a) Results of listing and ranking exercise by individual village } \\
\hline \multicolumn{2}{|c|}{ Ranked priority health issue } & \multicolumn{2}{|l|}{ Kangeme } & \multicolumn{3}{|c|}{ Ukumbisiganga } & Usinga \\
\hline \multicolumn{2}{|c|}{1} & \multicolumn{2}{|l|}{ Sleeping sickness } & \multicolumn{3}{|c|}{ Sleeping sickness } & Sleeping sickness \\
\hline \multicolumn{2}{|l|}{2} & \multicolumn{2}{|l|}{ Malaria } & \multicolumn{3}{|c|}{ Malaria } & Malaria \\
\hline \multicolumn{2}{|l|}{3} & \multicolumn{2}{|l|}{ Pneumonia } & \multicolumn{3}{|c|}{$\begin{array}{l}\text { Diarrhoea, with or without } \\
\text { blood }\end{array}$} & Bone pain/disease \\
\hline \multicolumn{2}{|l|}{4} & \multicolumn{2}{|c|}{ Bloody diarrhoea TB } & \multicolumn{3}{|l|}{$\mathrm{TB}$} & $\begin{array}{l}\text { Diarrhoea \& } \\
\text { vomiting TB }\end{array}$ \\
\hline \multicolumn{2}{|l|}{5} & \multicolumn{2}{|c|}{ Typhoid HIV/AIDS } & $\begin{array}{l}\text { Sore } \\
\text { Worn }\end{array}$ & Worms & & Vision problems \\
\hline \multicolumn{2}{|l|}{6} & \multicolumn{2}{|l|}{ Measles } & \multicolumn{3}{|c|}{$\mathrm{HIV} / \mathrm{AIDS}$} & Ear infections \\
\hline 7 & & \multicolumn{2}{|l|}{-} & \multicolumn{3}{|c|}{ Herpes } & Skin conditions \\
\hline 8 & & \multicolumn{2}{|l|}{-} & \multicolumn{3}{|c|}{ Skin conditions } & - \\
\hline \multicolumn{8}{|c|}{ b) Results of pair-wise ranking matrix, Kangeme village } \\
\hline & SS & Malaria & Mer & asles & TB & Skin disease & Vision problems \\
\hline Sleeping sickness (SS) & - & - & - & & - & - & - \\
\hline Malaria & SS & - & - & & - & - & - \\
\hline Measles & SS & Malaria & - & & - & - & - \\
\hline Tuberculosis (TB) & SS & Malaria & Mea & asles & - & - & - \\
\hline Skin disease & SS & Malaria & Mea & asles & TB & Skin disease & - \\
\hline Vision problems & SS & Malaria & & lecided & $\begin{array}{l}\text { Vision } \\
\text { probl }\end{array}$ & $\begin{array}{c}\text { Vision } \\
\text { ns } \\
\text { problems }\end{array}$ & - \\
\hline
\end{tabular}

When given the hypothetical scenario that any one of the previously prioritised diseases could be eliminated from their village, half the groups chose HAT and the other, malaria.

Several explanations were provided to explain why HAT was considered responsible for such a significant burden at community level, despite its relatively low incidence compared to other diseases. A commonly-cited explanation was the prolonged recovery phase for HAT, meaning that patients could often not resume normal daily activities (such as tilling the fields and collecting water or firewood) for 624 months. This results in a considerable loss of income and potentially devastating economic and social effects at household-level. In contrast, informants felt that whilst potentially an acute and severe disease, patients with malaria are typically fully recovered 'within weeks'.

"It is easier to die of malale ya ndorobo [sleeping sickness] whereas malaria will just delay you for a while."

"For the first month after treatment [for sleeping sickness] I was only able to earn half my usual income from carpentry and agriculture, but after three months I was able to earn my normal wage of TSh 2,000 per day again."
Additionally, the signs and symptoms of HAT were felt difficult to distinguish from malaria and other febrile illnesses, and confirmation of diagnosis and treatment was possible only by travelling considerable distances to KHC. Comparatively, villagers felt confident they could recognize the signs and symptoms of malaria and manage infection using anti-malaria drugs that they were able to purchase at local dispensaries.

While drugs for the treatment of HAT are provided free of charge at KHC, informants stated that other costs associated with treatment create a substantial economic burden for HAT patients and their families. Direct costs due to HAT include hospital fees, travel and living away from home expenses. In addition, prior to being diagnosed patients have often spent considerable amounts of money on clinical consultations, diagnostic procedures and/or drugs from local dispensaries. The most heavily-affected villages are located 40 $90 \mathrm{~km}$ from KHC and patients are typically accompanied by several relatives or friends, so that transport by train or bicycle is expensive and time consuming. Current treatment protocols require patients to remain in hospital on average for one month, when they are required to provide their own food and incidentals (e.g. drinks, meals, soap etc.) 
and to pay hospital fees of TSh1,000/day. Patients diagnosed with HAT in the past estimated that total direct costs associated with treatment were around TSh50,000-100,000; the equivalent of 1-2 months household income for many villagers in this area ${ }^{12,14}$. Many respondents found estimating a monetary figure for the indirect costs of HAT due to loss of income or productivity difficult, because many were dependent on subsistence farming. All respondents with a past diagnosis of HAT described loss of income/productivity for significant periods (including the time lived with disability prior to HAT diagnosis, during treatment and the extended recovery period) ranging on average from 5-24 months.

"Before sleeping sickness I used to harvest ten bags of maize, one bag of beans, five bags of rice and four bags of ground nuts per year, however, after sleeping sickness I only get two bags of ground nuts."

A variety of physical, psychological, and emotional consequences were also highlighted, including stress, fear, frustration, and feelings of dependency:

"There was a lot of emotional stress to my wife [when I had sleeping sickness]. We both had in our mind all those people who had died with a similar disease and we lost hope."

"Some people [sleeping sickness cases] can't return to their previous jobs, they may not recover normal mental functioning and might need someone to care for them."

Physical effects such as impotence, back and chest pain and mental impairment were described by several respondents previously treated for HAT and although there appeared to be no stigma specifically associated with HAT, respondents felt that stigma might arise due to these physical effects. Respondents also described symptoms they thought to be the side-effects of melarsoprol treatment including swelling of the body, skin irritation/rashes and back pain continuing for several months after treatment.

"I think the drug [melarsaprol] is very bad because the nurse kept asking me to talk to her and checking that I was still ok while she was administering it. I am not feeling completely better. The side effects of the treatment are bad, now although I can get an erection, I am not able to have sex because I am too weak. Even today I cannot do any work despite getting treatment. I cannot even ride a bicycle now. They need to try and make some drugs that are not so strong."
"I want to ask you, how come my health is not improving even after taking these drugs [melarsaprol]? Do you have any drugs in your bags that will give me strength back to my body? .... I have pain inside the nerves and the muscles. My muscles really hurt even when I turn my neck and back."

\section{Delays to effective diagnosis and treatment}

MoHSW and KHC informants concurred that it is extremely difficult to diagnose HAT clinically because first stage symptoms are indistinguishable from other more common febrile illnesses such as malaria,. Furthermore, the neurological symptoms of second stage HAT may be confused with cerebral malaria or tuberculosis. Informants hence stressed the importance of microscopic confirmation of suspected HAT by indentifying trypanosomes in a patient's blood or cerebrospinal fluid, but also recognized that access to confirmatory testing is severely limited for people living in affected communities in this setting, where village health workers are not equipped or trained to provide such diagnostic services. Parasite-based microscopic diagnosis of HAT and malaria is currently available only at larger health care facilities, such as KHC, whilst rapid diagnostic tests (RDTs) for malaria are not yet available in study villages and are yet to be developed for T.b. rhodesiense. Even where appropriate diagnostic facilities are available such as at KHC, delays in confirming HAT appear to be common according to testimonies from patients interviewed in the study.

During their initial medical consultations at $\mathrm{KHC}$, some patients have tested positive for other infections such as malaria or typhoid, which when combined with a false-negative HAT result lead to them being discharged and further delays in HAT diagnosis and treatment. If HAT is not detected in the blood, health care staff may be reluctant to carry out a lumbar puncture, required to check if the trypanosomes have progressed into the CSF, especially if an alternative diagnosis of malaria or typhoid is possible. This was exemplified in several case studies where patients reported that they had specifically requested a lumbar puncture be performed to confirm or rule-out HAT despite initial opposition from KHC staff who proposed treatment for alternate diagnoses.

Interviews with three village health workers (a dispensary nurse and two traditional healers), indicated that all felt a high level of confidence in 
their ability to make a clinical diagnosis of HAT and to discriminate HAT from other common febrile diseases. Traditional healers were reluctant to share the specifics of their diagnostic methods with the research team but the diagnostic process included "communing with the spirits who inform us of the diagnosis and treatment."

The dispensary nurse used separate lists to detail the clinical features of HAT and malaria and to differentially diagnose the two diseases. However, most of the symptoms and signs noted for HAT represented second stage disease, reiterating the difficulty of making a confident clinical diagnosis of early stage disease in the absence of confirmatory diagnostics. The dispensary nurse and traditional healers agreed that they were unable to treat HAT patients at community-level and that they referred all suspected cases to KHC.

Community representatives and stakeholders in study villages were asked about their perceptions, beliefs and health seeking behaviour related to febrile illness. At the onset of a febrile episode, malaria was typically suspected and anti-malarial drugs initiated using medication available in local shops and pharmacies. Persistence or recurrence of fever resulted in further rounds of self medication for suspected malaria, contributing to delays in HAT diagnosis and treatment.

\section{Knowledge, attitude and practices}

Community education programs and a history of trypanosomiasis in the area are thought to have contributed to the high level of knowledge of HAT and the role that tsetse flies play in transmission. Biomedical frameworks of health and disease causation appeared widely accepted in the villages. It was also thought that community education programs miss those who spend large amounts of time outside the village, such as the semi-nomadic Sukuma cattle-herders. A cattle-herder in the area at the time of the study stated that while he had been informed about animal trypanosomiasis from the District veterinary officer and treated his cattle for trypanosomiasis, he had not been informed that certain species of trypanosomes could infect, and be fatal to humans. This situation exemplified the disjunction between the management and treatment of animal (coordinated by MoLDF) and human trypanosomiasis (coordinated by MoHSW) in Urambo, despite strong evidence supporting their inter-relatedness ${ }^{15-18}$.

\section{Transmission and risk characteristics}

The villages at greatest risk within Urambo district are all located in close proximity to the vegetated north-western part of Ugala Game Reserve. When driving through this stretch of forest it is clear that tsetse flies are ubiquitous and those not protected will inevitably be bitten. However, there are mixed opinions within Urambo District as to which individuals are at greatest risk. Government officials and community stakeholders believed that increased contact with tsetse flies increases the risk of disease acquisition and that individuals whose occupations are carried out in the forest (honey gathering, timbering, fishing etc) are at greatest risk. For example, timber men and honey gatherers estimated that they are bitten around 20-150 times perday: "I go honey-gathering in the forest 2-3 times in MayOctober and stay there for 1 month each time. I get 20-30 bites per day in the forest and 3 bites per day in the agricultural fields."

"In the past I used to stay in the forest areas gathering honey and I was constantly bitten. I stopped honey gathering in 2003"

A number of villagers however stated that they had seen just as many HAT cases among those without such occupational risks. Five of the six HAT patients interviewed during the study reported minimal activities in forested areas and believed they had contracted HAT collecting firewood or carrying out subsistence farming, where they also estimated that they were typically bitten $<10$ times perday. One case could not recall being bitten at all. The majority of community-based key informants considered all occupations to be at risk, reasoning that people could be bitten in the village, in agricultural fields or when venturing into forest areas to gather essential items such as honey, fire-wood or water. Villagers also stated that the risk of contracting HAT is present throughout the year while malaria is seasonal, and commented that it is more difficult to protect one self against tsetse flies than mosquitoes.

Participatory community mapping was particularly effective in understanding transmission and risk characteristics. Areas heavily infested with tsetse flies could be found in close proximity to the villages with these same areas frequented by villagers for purposes of collecting water and firewood and tilling fields. Whilst each village was bordered by a 
handful of tsetse fly targets these were not sufficient in controlling the vector.

\section{Discussion}

Hospital records from KHC provided crude case numbers and validated the focal nature of the disease's resurgence in Urambo district, northwest Tanzania. Attempts were made to explain the trends in case numbers but from the relatively small case numbers, limited time series and myriad of explanations offered, these findings need to be interpreted with caution. The estimated level of under-reporting was consistent with a recent study conducted in Uganda ${ }^{19}$, where only an estimated $60 \%$ of all HAT cases in the study area were reported. More detailed hospital and peripheral health facility records could provide essential epidemiological data and be an important advocacy tool. Of the hospitalreported fatalities, it was not documented whether death was due to drug-related toxicity or an outcome of the disease itself.

Given the poor prognosis of stage two disease $^{20}$, there is a strong argument for earlier diagnosis and treatment at local level ${ }^{21}$ and at international level, a clear need to continue the search for less toxic drug regimes for advanced disease. New second stage drugs are currently in development ${ }^{22-23}$ but are unlikely to become available in the near future, so that reducing the burden of HAT in Urambo in the short-medium term will require a clear commitment to improving access to diagnostic and treatment services. The development of Loop-Mediated Isothermal Amplification (LAMP) diagnostic tests for $\mathrm{HAT}^{24}$, suitable for use in village health facilities, has the potential to improve delays in diagnosis. In their absence, the most immediate means of improving such delays is by being able to quickly rule out other common infections such as malaria with the use of currently available RDTs for malaria.

At the time of the study, WHO, NIMR and KHC were collaborating to provide a microscope and the necessary training for staff at Ukumbisiganga Dispensary. This would allow residents from surrounding villages including Lumbe, Usinga and Kangeme to be tested for HAT at their local dispensary, avoiding a long journey to Kaliua and unnecessary costs to patients. This capability, however, will not include the ability to centrifuge the sample prior to microscopy, reducing its sensitivity ${ }^{23}$. Additionally, as stage two diagnosis requires suramin administration prior to lumbar African Health Sciences Vol 12 No 2 June 2012 puncture (unavailable at Ukumbisiganga Dispensary), diagnosis at community-level will be limited to stage one disease. Microscopy will also be used to exclude or confirm malaria infection and help avoid unnecessary repeat treatments for suspected malaria. If high-quality local laboratory diagnostic services can be sustained, and used in conjunction with those at KHC, patients should see a reduction in diagnostic delays for HAT. Access to diagnostic services could also be improved by enabling suspected HAT cases to travel for free to KHC via the government-owned railway system, as previously implemented during an earlier study conducted at KHC. Facilitating free travel would also have an added benefit in promoting early presentation prior to the onset of advanced disease.

While the use of DALYs are fundamental in providing a quantitative assessment of disease burden and thus prioritizing limited public health resources, they are unable to fully capture the impact a disease has on individuals, their families and the wider community. For HAT in Urambo District the DALYs (age adjusted and accounting for $45 \%$ underreporting) were calculated to be $978.9^{8}$ - a metric which says little about the lived experience of the disease in high risk-communities and among affected individuals. Qualitative approaches can allow for a more complete understanding of the impact of a disease in the community, and the complexities involved in controlling the disease and improving patient outcomes. Triangulation of data from participatory research activities and KII's clearly indicates that community members consider the burden of HAT to be significant, and comparable to that of malaria, in affected villages. Village-level and MoHSW stakeholders differed in their perception of the local HAT disease burden, a gap that represents an important barrier to effectively tackling HAT in Urambo and potentially in other affected rural areas in Tanzania. Local and nationallevel advocacy and awareness campaigns will be required to close these gaps.

The epidemiology of HAT in Urambo remains unclear and will be essential to clarify in order to develop more effective and accessible diagnostic, treatment and control programs. Analyzing HAT data by place of infection on a longitudinal basis would provide valuable data on local risk and transmission characteristics and indicate whether control programs were effective. Without an accurate picture of the true epidemiology of HAT in Urambo, associations with environmental, 
vector, animal, and human factors cannot be analyzed and used to inform policy.

Including those living in the most affected communities in the design and implementation of locally-appropriate interventions for HAT will be crucial to the success of future interventions, and to reducing the local burden of this important neglected disease. For example, designing appropriate riskreduction strategies in partnership with community stakeholders would facilitate efficient and effective program design. Asking villagers to reduce time spent in and around heavily tsetse-infested forest areas is unlikely to be feasible given communities' critical dependence on the land for their largely subsistence economy. The utility and acceptability of a variety of alternative approaches could be explored through such a participatory planning process, including the feasibility of using permethrin-impregnated clothing when entering the forest ${ }^{25}$; using the most costeffective and efficient visual bait tsetse traps ${ }^{26}$; working with community stakeholders to ensure the most appropriate placement of visual baits e.g. in relation to established honey and timber gathering sites; and the role of periodic aerial insecticide spraying, as employed very effectively in Botswana's Okavango Delta ${ }^{27}$.

\section{Conclusion}

Assessing the burden of a suspected resurgence in HAT in three villages in Urambo district Tanzania, exemplified many of the difficulties in capturing health-related information in a poor rural setting. The use of participatory tools enabled communities to articulate their lived experience of HAT and the burden that they felt the disease placed on their lives. The involvement of stakeholders at all levels demonstrated the disparity between primary and secondary stakeholders in perceptions of local disease burden. Adopting a participatory mixedmethods approach provided an effective and efficient means to investigate the impact of this neglected disease in an isolated, resource-limited context, and provided valuable results to inform future policy and operations research priorities.

\section{Acknowledgements}

We are extremely grateful for the expertise of the Tanzanian team who helped to undertake this survey and would like to thank all the participants involved, without whom the work would not have been possible.

\section{References}

1. Kennedy PGE. Human African trypansomiais of the CNS: current issues and challenges. The Journal of Clinical Investigation. 2004;113(4):496504.

2. Murray CJL, Lopez AD. The Global Burden of Disease: a comprehensive assessment of mortality and disability from diseases, injuries and risk factors in 1990 and projected to 2020. Cambridge: Harvard University Press; 1996.

3. Fevre EM, Wissmann BV, Welburn SC, Lutumba P. The Burden of Human African Trypanosomiasis. PLoS Neglected Tropical Diseases. 2008 12/23;2(12):e333.

4. Odiit M, Shaw A, Welburn SC, egrave, vre EM, Coleman PG, et al. Assessing the patterns of health-seeking behaviour and awareness among sleeping-sickness patients in eastern Uganda. Annals of Tropical Medicine and Parasitology. 2004;98:339-48.

5. Lutumba P, Makieya E, Shaw A, Meheus F, Boelaret M. Human African trypanosomiasis in a rural community, Democratic Republic of Congo. Emerging Infectious Diseases. 2007;13(2):24854.

6. Kabayo JP. Aiming to eliminate tsetse from Africa. Trends in Parasitology. 2002;18(11):473-5.

7. WHO, editor. Pan African tsetse and trypanosomiasis eradication campaign: Report by the Secretariat. Fifty-Fifth World Health Assembly; 2002; Geneva, Switzerland.: World Health Organization.

8. Matemba LE, Fèvre EM, Kibona SN, Picozzi K, Cleaveland S, Shaw AP, et al. Quantifying the burden of rhodesiense sleeping sickness in Urambo District, Tanzania. PLoS Neglected Tropical Diseases 2010;4(11).

9. IIED. Participartory Learning and Action Notes: Decentralisation and community-based planning: International Institute for Environment and Development (IIED) 2004 Contract No.: 49.

10. Hansen EC. Successful qualitative health research: a practical introduction. Crows Nest, Australia: Allen and Unwin; 2006.

11. NBS. 2002 Population and Housing Census: Tanzania National Bureau of Statistics2002.

12. Sindato C. Factors influencing individual and community participation in the control of tsetse flies and human African trypanosomiasis in Urambo District, Tanzania. Tanzania Journal of Health Research. 2008;10. 
13. WHO. Roll Back Malaria Monitoring and Evaluation: World Health Organisation 2005.

14. UNDP. Human Development Report 2007/08: United National Development Program2008.

15. Hide G, Maudlin TI, Welburn SC. The origins, dynamics and generation of Trypanosoma brucei rrhodesiense epidemics in East Africa. Parasitology Today. 1996;12(2):50-5.

16. Fevre EM, Coleman PG, Oditt M, Magona JW, Welburn SC, Woolhouse MEJ. The origins of a new Trypanosoma brucei rhodesiense sleeping sickness outbreak in eastern Uganda. Lancet. 2001;358:625-28.

17. Kuzoe FAS, Schofield CJ. Strategic review of traps and targets for tsetse and African trypanosomiaisis control: UNICEF/UNDP/ World Bank/WHO Special Programme for Research and Training in Tropical Diseases2004.

18. Batchelor NA, Atkinson PM, Gething PW, Picozzi K, Fèvre EM, Kakembo ASL, et al. Spatial Predictions of Rhodesian Human African Trypanosomiasis (Sleeping Sickness) Prevalence in Kaberamaido and Dokolo, Two Newly Affected Districts of Uganda. PLoS Negl Trop Dis. 2009;3(12):e563.

19. Odiit M, Coleman PG, Liu W-C, McDermott JJ, Fevre EM, Welburn SC, et al. Quantifying the level of under-detection of Trypanosoma brucei rhodesiense sleeping sickness cases. Tropical Medicine and International Health. 2005;10(9):840-9.

20. Kennedy PGE. Human African trypanosomiasis of the CNS: current issues and challenges. Journal of Clinical Investigation. 2004;113(4):496-504.
21. Bukachi SA, Wandibba S, Nyamongo IK. The treatment pathways followed by cases of human African trypanosomiasis in western Kenya and eastern Uganda. Annals of Tropical Medicine and Parasitology. 2009;103:211-20.

22. Vodnala SK, Ferella M, Lundén-Miguel H, Betha E, van Reet N, Amin DN, et al. Preclinical Assessment of the Treatment of Second-Stage African Trypanosomiasis with Cordycepin and Deoxycoformycin. PLoS Negl Trop Dis. 2009;3(8):e495.

23. WHO. Clinical Enrolment and Collection Protocol: World Health Organisation2007.

24. Njiru ZK, Mikosza ASJ, Armstrong T, Enyaru JC, Ndung'u JM, Thompson ARC. LoopMediated Isothermal Amplification (LAMP) Method for Rapid Detection of Trypanosoma brucei rhodesiense. PLoS Negl Trop Dis. 2008;2(2):e147.

25. Sholdt LL, Schreck CE, Mwangelwa MI, Nondo J, Siachinji VJ. Evaluations of permethrinimpregnated clothing and three topical repellent formulations of deet against tsetse flies in Zambia. Med Vet Entomol. 1989 Apr;3(2):153-8.

26. Lindh JM, Torr SJ, Vale GA, Lehane MJ. Improving the cost-effectiveness of artificial visual baits for controlling the tsetse fly Glossina fuscipes fuscipes. PLoS Negl Trop Dis. 2009;3(7):e474.

27. Kgori PM, Modo S, Torr SJ. The use of aerial spraying to eliminate tsetse from the Okavango Delta of Botswana. Acta Trop. 2006 Oct;99(23):184-99. 